\title{
Finite-volume correction on the hadronic vacuum polarization contribution to the muon $g-2$ in lattice QCD
}

\author{
Taku Izubuchi, ${ }^{1,2}$ Yoshinobu Kuramashi, ${ }^{3,4}$ Christoph Lehner, ${ }^{2}$ and Eigo Shintani ${ }^{4}$
}

(PACS Collaboration)

${ }^{1}$ RIKEN-BNL Research Center, Brookhaven National Laboratory, Upton, New York 11973, USA

${ }^{2}$ Physics Department, Brookhaven National Laboratory, Upton, New York 11973, USA

${ }^{3}$ Center for Computational Sciences, University of Tsukuba, Tsukuba, Ibaraki 305-8577, Japan ${ }^{4}$ RIKEN Center for Computational Science, Kobe, Hyogo 650-0047, Japan

(Received 24 May 2018; published 6 September 2018)

\begin{abstract}
We study the finite-volume correction on the hadronic vacuum polarization contribution to the muon $g-2\left(a_{\mu}^{\text {hvp }}\right)$ in lattice QCD at (near) physical pion mass using two different volumes: $(5.4 \mathrm{fm})^{4}$ and $(8.1 \mathrm{fm})^{4}$. We use an optimized AMA technique for noise reduction on $N_{f}=2+1$ PACS gauge configurations with stout-smeared clover-Wilson fermion action and Iwasaki gauge action at a single lattice cut-off $a^{-1}=2.33 \mathrm{GeV}$. The calculation is performed for the quark-connected light-quark contribution in the isospin symmetric limit. We take into account the effects of backward state propagation by extending a temporal boundary condition. In addition, we study a quark-mass correction to tune to the exact same physical pion mass on different volume and compare those correction with chiral perturbation. We find $10(26) \times 10^{-10}$ difference for light quark $a_{\mu}^{\text {hvp }}$ between $(5.4 \mathrm{fm})^{4}$ and $(8.1 \mathrm{fm})^{4}$ lattice in $146 \mathrm{MeV}$ pion.
\end{abstract}

DOI: 10.1103/PhysRevD.98.054505

\section{INTRODUCTION}

The muon anomalous magnetic moment $(g-2)$ is an essential observable for a rigorous test of the standard model (SM) of particle physics. The experimental value of the muon $g-2, a_{\mu}^{\mathrm{E} 821}=11659209.1(5.4)(3.3) \times 10^{-10}$, has been measured more than a decade ago at BNL in the E821 experiment $[1,2]$ and currently exhibits a $3 \sigma-4 \sigma$ tension with the SM theory prediction. Within the theory prediction, the QED corrections are now known to fiveloop order [3] surpassing the precision of $a_{\mu}^{(\mathrm{E} 821)}$ by 2 orders of magnitude. The electroweak interaction contribution involving $W^{ \pm}, Z$, and the Higgs is known 1 order of magnitude more precisely than $a_{\mu}^{\mathrm{E} 821}$. The theory uncertainty is currently dominated by the leading-order QCD contribution, i.e., the hadronic vacuum polarization (HVP) contribution. This contribution is typically extracted from $e^{+} e^{-} \rightarrow$ hadron [4-7] or $\tau \rightarrow$ hadron decays [8-11] using a dispersion relation [12-15] and currently has an uncertainty similar to the error of $a_{\mu}^{\mathrm{E} 821}$. A comparable uncertainty

Published by the American Physical Society under the terms of the Creative Commons Attribution 4.0 International license. Further distribution of this work must maintain attribution to the author(s) and the published article's title, journal citation, and DOI. Funded by SCOAP ${ }^{3}$. comes from the hadronic light-by-light contribution whose model dependence is still under scrutiny [16]. To resolve the discrepancy between experiment and the SM calculation we need to reduce the uncertainties of both QCD contributions and the experiment. The upcoming experiments at Fermilab [17] and J-PARC [18] aim for a fourfold improvement over $a_{\mu}^{\mathrm{E} 821}$ in the near future which makes a similar precision improvement of the HVP contribution of timely interest.

The current estimate of the leading order of the hadronic contribution (HLO) to muon $g-2$ is $a_{\mu}^{\mathrm{HLO}}=(693.3(2.5) \times$ $10^{-10}$ for $e^{+} e^{-}$collision data and $688.9(3.5) \times 10^{-10}$ for $\tau$-decay data quoted from $[15,19,20]$. The determination from $e^{+} e^{-} \rightarrow$ hadrons $(\gamma)$ cross sections [21] as a function of center-of-mass energy includes the QED effect in the hadron vertex and radiative correction of the final state [19]. On the other hand, lattice QCD, which is a rigorous computation from the first principle of QCD, is able to provide the pure QCD contribution to $a_{\mu}^{\text {hvp }}$ for the whole energy region, and its calculation is completely independent from the dispersive approach. Therefore a high-precision lattice QCD result is required for a cross-check of and potential improvement over the dispersive value.

Recently the lattice QCD community has made significant progress to improve the precision of $a_{\mu}^{\text {hvp }}$. Starting from quenched QCD calculations [22,23], $N_{f}=2$ or $2+1$ 
QCD calculations using various lattice fermion formulation and lattice parameters have been reported by several groups [24-32] and recent lattice QCD calculations with a perturbative QED correction at the physical pion mass is now available [33]. Currently, however, the precision of pure lattice calculations is about 5 times lower than of the dispersive approach. One major source of uncertainty in a lattice QCD evaluation of $a_{\mu}^{\text {hvp }}$ is the statistical noise of the Monte Carlo method. In addition, finite-volume (FV) corrections for lattice size $L \sim 5-6 \mathrm{fm}$ have been expected to be significant (see, e.g., [33]) and it then has been so far only treated by an effective-field theory [34,35]. An estimate of the FV correction with the pure lattice calculation is therefore highly desired to control systematic error in a lattice calculation for the desired precision.

In this paper, to study $\mathrm{FV}$ correction to $a_{\mu}^{\text {hvp }}$ in purely lattice QCD, we compare the connected HVP diagram between two volumes, $L=5.4$ and $8.1 \mathrm{fm}$, at nearly physical pion $\left(m_{\pi} \simeq 0.14 \mathrm{GeV}\right)$, which are corresponding to two variations of $m_{\pi} L=3.8$ and 5.8. Since the statistical noise of the infrared region should be significantly reduced to perform a rigorous test of FV effect, we utilize a highly optimized all-mode-averaging (AMA) technique reported in [36] which can further improve the performance rather than the original proposals [37-39]. Our study also provides a test of the usage of an effective-field theory for the FV correction as used in $[29,31,33]$ and a crosscheck from pure lattice calculation.

This paper is organized as the followings. In Sec. II, we introduce the method to compute $a_{\mu}^{\text {hvp }}$. Section III shows our setup for the numerical computation, and in Sec. IV we present numerical results on two different volumes. In Sec. V, we discuss mass correction and the FV effect obtained by our numerical study. Finally, in Sec. VI, we summarize this paper and discuss future extensions.

\section{LATTICE COMPUTATION OF $\boldsymbol{a}_{\boldsymbol{\mu}}^{\mathrm{hvp}}$}

Since the lattice QCD calculation is defined in Euclidean space-time, a conventional representation of $a_{\mu}^{\text {hvp }}$ is as the integral of the vacuum polarization function $\Pi(Q)$ with respect to the Euclidean momentum squared $Q^{2}$ from zero to infinity,

$$
\begin{gathered}
a_{\mu}^{\mathrm{hvp}}=\left(\frac{\alpha_{e}}{\pi}\right)^{2} \int_{0}^{\infty} d Q^{2} K_{E}\left(Q^{2}\right) \hat{\Pi}\left(Q^{2}\right), \\
\hat{\Pi}\left(Q^{2}\right) \equiv \Pi\left(Q^{2}\right)-\Pi(0), \\
K_{E}(s)=\frac{1}{m_{\mu}^{2}} \hat{s} Z^{3}(\hat{s}) \frac{1-\hat{s} Z(\hat{s})}{1+\hat{s} Z^{2}(\hat{s})}, \\
Z(\hat{s})=-\frac{\hat{s}-\sqrt{\hat{s}^{2}+4 \hat{s}}}{2 \hat{s}}, \quad \hat{s}=\frac{s}{m_{\mu}^{2}},
\end{gathered}
$$

which can be derived by analytic continuation from the original representation using a timelike momentum $q^{2}\left(=-Q^{2}\right)[22,40] . K_{E}$ is a known QED kernel resulting from the needed one-loop computation and $\alpha_{e}$ is a fine structure constant $\alpha_{e}=1 / 137.03599914$. $\hat{\Pi}$ denotes the subtracted vacuum polarization function at $Q^{2}=0$. For the lattice computation of $a_{\mu}^{\text {hvp }}$, due to the nonzero lattice spacing and finite volume, we convert its integral to a finite momentum sum. In this paper we deal with the integral in Eq. (1) as the coordinate space-time summation of vectorvector current correlators on the lattice, in the so-called "time-momentum representation" (TMR) [41].

We use the vector-vector current correlator at zero momentum in spatial direction $i$,

$$
C(t)=\frac{1}{3} \sum_{i=1}^{3} \int d^{3} \vec{x}\left\langle V_{i}^{\mathrm{cv}}(\vec{x}, t) V_{i}^{\mathrm{loc}}(0)\right\rangle
$$

with local lattice current

$$
V_{\mu}^{\mathrm{loc}}=Z_{V} \bar{q}(x) \gamma_{\mu} q(x)
$$

and $Z$ factor $Z_{V}=0.95153(76)(1487)$, evaluated by the Schrödinger functional method [42]. We also use the conserved current

$$
\begin{aligned}
V_{\mu}^{\mathrm{cv}}(x)= & \frac{1}{2}\left[\bar{q}(x+a \hat{\mu})\left(1+\gamma_{\mu}\right) U_{\mu}^{\dagger}(x) q(x)\right. \\
& \left.-\bar{q}(x)\left(1-\gamma_{\mu}\right) U_{\mu}(x) q(x+a \hat{\mu})\right],
\end{aligned}
$$

i.e., the point-split current that satisfies the Ward-Takahashi identity as also used in $[25,32,43]$. In the TMR, $a_{\mu}^{\text {hvp }}$ in Eq. (1) can be also represented as

$$
\begin{gathered}
a_{\mu}^{\mathrm{hvp}}=4 \alpha_{e}^{2} m_{\mu} \int_{0}^{\infty} d t t^{3} C(t) \tilde{K}(t), \\
\tilde{K}(t)=\frac{2}{m_{\mu} t^{3}} \int_{0}^{\infty} \frac{d \omega}{\omega} K_{E}\left(\omega^{2}\right)\left[\omega^{2} t^{2}-4 \sin ^{2}(\omega t / 2)\right],
\end{gathered}
$$

as shown in [41]. On the lattice, the above becomes the summation of discretized $C(t)$ multiplied with $\tilde{K}(t)$ up to a half-length of lattice temporal extension. ${ }^{1}$ Setting the truncation bound of the sum to $t_{\text {cut }}<N_{t} a / 2$ in an integral of Eq. (7), the lattice representation is

$$
\left[a_{\mu}^{\mathrm{hvp}}\right]_{\mathrm{lat}}\left(t_{\mathrm{cut}}\right)=\frac{1}{2} \sum_{t / a=0}^{t_{\mathrm{cut}} / a-1}\left[C(t) W_{t}(t)+C(t+a) W_{t}(t+a)\right],
$$

\footnotetext{
${ }^{1}$ In periodic or antiperiodic boundary conditions, BSP significantly alters the $C(t)$ at $t \sim N_{t} / 2 a$, which is one of the FV (or finite temporal extension) effects. We numerically study this in Sec. IV.
} 
$W_{t}(t)=8 \alpha_{e}^{2} \int_{0}^{\infty} \frac{d \omega}{\omega} K_{E}\left(\omega^{2}\right)\left[\omega^{2} t^{2}-4 \sin ^{2}(\omega t / 2)\right]$,

in which the expression of $\tilde{K}$ in Eq. (8) is substituted and the trapezoidal formula is used for numerical integral.

We also note that the representation of Eq. (9) is not unique for finite lattice spacing. For example, if we use the sin functional form of lattice momentum, $\tilde{Q}=2 a^{-1}$ $\sin \left(Q_{\mu} / 2 a\right)$, such a representation is changed to

$$
\left[\tilde{a}_{\mu}^{\mathrm{hvp}}\right]_{\mathrm{lat}}\left(t_{\mathrm{cut}}\right)=\frac{1}{2} \sum_{t / a=0}^{t_{\mathrm{cut}} / a-1}[C(t) \tilde{W}(t)+C(t+a) \tilde{W}(t+a)],
$$

$\tilde{W}(t)=8 \alpha_{e}^{2} \int_{0}^{\infty} \frac{\omega d \omega}{\tilde{\omega}^{2}} K_{E}\left(\omega^{2}\right)\left[\tilde{\omega}^{2} t^{2}-4 \sin ^{2}(\omega t / 2)\right]$,

where we use $\tilde{\omega}=2 a^{-1} \sin (a Q / 2)$. The trivial difference between Eqs. (9) and (11) is at $t=a$, in which the integrand of $\left[\tilde{a}_{\mu}^{\text {hvp }}\right]_{\text {lat }}$ is zero, besides that of $\left[a_{\mu}^{\text {hvp }}\right]_{\text {lat }}$ is nonzero. The difference between $\left[a_{\mu}^{\text {hvp }}\right]_{\text {lat }}$ and $\left[\tilde{a}_{\mu}^{\text {hvp }}\right]_{\text {lat }}$ can be used as a simple estimate of lattice artifacts (see Sec. VI).

\section{A. Strategy to measure finite-volume effect}

In this paper, we numerically estimate the FV correction in the TMR directly at physical pion mass by comparing two volumes at the same cutoff scale. This allows us to remove uncertainties due to the chiral extrapolation ansatz [44], which becomes the large contribution around the physical pion extrapolated from an unphysically heavy mass [32]. As we will see, pion masses on the two volumes are both very close to the physical mass, but there is a small difference between the two masses [45]. To clearly separate the FV effect from the effects from using a slightly different pion mass, we correct this small mass difference by adjusting the valence light-quark mass as well as the sea quark mass via the reweighting technique [46].

One practical issue in using the TMR is that in order to evaluate the $t$-integral one needs to precisely evaluate vector-vector current correlators at a large distance before the integrand at the infrared regime is negligibly small. This is a concern since the lattice data is limited to $|t|<N_{t} a / 2$ and the statistical noise grows exponentially with time.
One idea to carry out such an integral (time-slice summation) at a large distance is to model the correlation function by the multi-hadron state ansatz or parametrizations of rho meson decay $[29,32,41]$. The assumptions made may be more accurate when the pion mass is unphysically heavy or the size of the lattice box is small, but it is not clear how reliable these models are at physically light-quark mass and large volume in which the rho meson becomes unstable and two-pion states become more dominant.

In addition, there are two different kinds of effects due to the finite four-dimensional volume. One effect is from the finite extent in the temporal direction, which causes the backward state propagation (BSP) due to the periodic boundary condition in time. Another more complicated effect is the finite spatial volume effect. To compare results in two different spatial volumes for a large enough time extent, so that the BSP effect is exponentially suppressed and becomes negligible, we extended the time extent $N_{t}$ of a gauge configuration, $U_{\mu}(x, t)$, by a factor of $\mathrm{w}$ by concatenating two identical lattices together in the time direction,

$$
U_{\mu}^{\mathrm{ext}}(x, t)=\left\{\begin{array}{ll}
U_{\mu}(x, t), & \left(0 \leq t / a<N_{t}\right) \\
U_{\mu}\left(x, t-N_{t}\right), & \left(N_{t} \leq t / a<2 N_{t}-1\right)
\end{array} .\right.
$$

which is identical to the utilization of combining the quark propagators with periodic and antiperiodic boundary conditions in the temporal direction onto the vector-vector current correlator. By comparing the $t$-integral on the original lattice, $U_{\mu}(x, t)$, and on the extended one, $U_{\mu}^{\mathrm{ext}}(x, t)$, we will observe a significant effect of the BSP contribution to $a_{\mu}^{\text {hvp }}$.

\section{LATTICE SETUP AND ITS PARAMETER}

In this paper, we use gauge configurations of a stout smeared non-perturbatively $\mathcal{O}(a)$ improved Wilson fermion in $N_{f}=2+1$ on Iwasaki gauge action with $\beta=1.82$ at the physical point (see Table I). The PACS Collaboration has generated it on two different volumes $L / a=64$ and 96, corresponding to $5.4 \mathrm{fm}^{4}$ and $8.1 \mathrm{fm}^{3}$, at a cutoff scale $a^{-1}=2.332(18)[42,47]$.

In the measurement of the vector-vector current correlator, we apply the AMA technique [37-39] to boost the statistical accuracy. AMA is defined with the master

TABLE I. Table of parameters of PACS gauge ensembles. $L$ denotes spatial length and $T$ denotes temporal length. In the Wilsonclover fermion, $K_{l}$ and $K_{s}$ denote the kappa value for light (up and down) quark flavor and strange quark flavor, respectively.

\begin{tabular}{lccccccc}
\hline \hline$L / a$ & $T / a$ & $K_{l}$ & $K_{s}$ & $m_{\pi}(\mathrm{GeV})$ & $m_{K}(\mathrm{GeV})$ & $m_{\pi} L$ & configurations \\
\hline $96[8.1 \mathrm{fm}]$ & $96[8.1 \mathrm{fm}]$ & 0.126117 & 0.124790 & $0.1461(4)$ & $0.5242(3)$ & 6.0 & 50 \\
$64[5.4 \mathrm{fm}]$ & $64[5.4 \mathrm{fm}]$ & 0.126117 & 0.124902 & $0.1385(9)$ & $0.5004(4)$ & 3.9 & 187 \\
& & $0.126119^{\mathrm{a}}$ & 0.124902 & $0.1354(9)$ & $0.4999(4)$ & 3.7 & 87 \\
\hline \hline
\end{tabular}

${ }^{\text {a }}$ This is a kappa for the valence quark to shift the pion mass to be $0.135 \mathrm{GeV}$. For the sea quark, we use the reweighting method to be on the unitary point. 
TABLE II. Table of parameters in our analysis using AMA on each ensemble. "Domain block" is a size of the domain in SAP. $n_{\text {cy }}$ is a number of the cycle of SAP. $N_{s}$ denotes the number of deflation vectors. The "Stop Iteration" column is a fixed number of the iteration of GCR with SAP deflation. $r_{\mathrm{src}}^{\min }$ denotes the minimum separation of a source point between $O^{\text {(appx) } g}$. The "Measurement" column is a total number of measurements for $O^{\text {(AMA) }}$, which consists of the number of configuration times $N_{G}$ measurements of $O^{(\text {appx })}$. The last two rows are in the ensemble whose temporal length is extended into the double size by duplication.

\begin{tabular}{|c|c|c|c|c|c|c|c|c|}
\hline$L / a$ & $T / a$ & Quark & Domain Block & $n_{\mathrm{cy}}$ & $N_{s}$ & Iteration & $r_{\mathrm{src}}^{\min } \mathrm{fm}$ & Measurement \\
\hline \multirow[t]{2}{*}{$96[8.1 \mathrm{fm}]$} & $96[8.1 \mathrm{fm}]$ & Light & $6^{4}$ & 5 & 40 & 5 & 1.43 & 102,141 \\
\hline & & Strange & & & 30 & & 2.03 & 3,382 \\
\hline \multirow[t]{4}{*}{$64[5.4 \mathrm{fm}]$} & $64[5.4 \mathrm{fm}]$ & Light & $4^{4}$ & 5 & 30 & 5 & 0.68 & 409,966 \\
\hline & & Strange & & & & & 1.35 & 6,247 \\
\hline & $128[10.8 \mathrm{fm}]$ & Light & & & & & 0.68 & 157,250 \\
\hline & & Strange & & & & & 2.71 & 1,376 \\
\hline \multirow[t]{2}{*}{$64[5.4 \mathrm{fm}]$} & $64[5.4 \mathrm{fm}]$ & $\begin{array}{c}\text { Light } \\
\text { (Reweight) }\end{array}$ & $4^{4}$ & 5 & 30 & 5 & 0.68 & 425,991 \\
\hline & $128[10.8 \mathrm{fm}]$ & $\begin{array}{c}\text { Light } \\
\text { (Reweight) }\end{array}$ & & & & & & 111,360 \\
\hline
\end{tabular}

formula for the measurement of target observable $O$, which is a vector-vector current correlator in this case,

$O^{\mathrm{AMA}}=\frac{1}{N_{\text {org }}} \sum_{f \in G}^{N_{\text {org }}}\left[O^{(\mathrm{org}) f}-O^{(\mathrm{appx}) f}\right]+\frac{1}{N_{G}} \sum_{g \in G}^{N_{G}} O^{(\mathrm{appx}) g}$,

with covariant transformation $g \in G$ under a subset of its symmetry $G$. Here $G$ corresponds to the translational symmetry and its size is $N_{G}$ for approximation and $N_{\text {org }}$ for original. In [36], one of the authors has developed the highly optimized AMA using Schwartz alternative procedure (SAP) deflation preconditioning [48,49]. From a knowledge of tuning parameters to reduce the computational cost of approximation in AMA, SAP deflation makes an achievement of the high performance in the measurement on PACS configurations compared to low-mode deflation (see the Appendix). Since for this study we need highly accurate lattice data of the vector-vector current correlator in the infrared region, we tune the parameter to generate the deflation field more efficiently in the large time slice. Using the limited number of gauge configurations to $<200$, separating 10 (20) and 40 HMC trajectory in $64^{4}\left(64^{3} \times 128\right)$ and $96^{4}$ lattice ensembles, respectively, we measure the approximation $O^{(\text {appx }) \mathrm{g}}$ with the $N_{G} \sim$ $\mathcal{O}\left(10^{3}\right)$ as different source points, and its total statistics is thus $\mathcal{O}\left(10^{6}\right)$ that we can achieve. Note that in the error analysis we use the 5 (2) jackknife bin size for the $64^{4}$ $\left(64^{4} \times 128\right)$ lattice ensemble, in which autocorrelation is small when using more than $40 \mathrm{HMC}$ trajectory in PACS configurations [47]. From the practical point of view, aiming for $N_{G} \sim \mathcal{O}\left(10^{3}\right)$, we tune the parameter of approximation to be small $\Delta r$ [37-39], in which $O^{(\text {appx) }}$ and $O^{(\text {org })}$ are strongly correlated, as $\Delta r \lesssim N_{G} / 2 \sim \mathcal{O}\left(10^{-4}\right)$ for the scaling of the statistical error close to $1 / \sqrt{N_{G}}$.
In Table II, we show the detail of parameters to generate an approximation $O^{(\text {appx) }}$ in AMA on each gauge ensemble. In the computation of $O^{\mathrm{AMA}}$, we use a method with a fixed number of iterations of the general conjugate residual (GCR) solver with SAP deflation as used in [36]. In a generation of the SAP deflation field, the domain block size, the number of the SAP cycle $n_{\text {cy }}$ and the number of theh deflation vector are tuned as in Table II. SAP is used in not only the preconditioning of GCR, but also in the generation of the deflation field overlapping with lowmode dominance by the smoothing technique (inexact deflation [49]).

\section{NUMERICAL RESULTS}

\section{A. Analysis of the vector-vector current correlator}

First, we show the time-separation dependence of the vector current two-point correlation function, $C(t)$, from short to long distance in Fig. 1. For the computation of $a_{\mu}^{\text {hvp }}$ using Eq. (9), we need to know the precise value of $C(t)$ in the large $t$ region. Our high-statistics result boosted by AMA method show a statistically significant signal beyond $t=2.7 \mathrm{fm}$, which is the longest temporal separation for our smaller $64^{4}$ lattice, and it thus allows us to compare large $t$ behavior of $C(t)$ on different volumes.

It is also noteworthy that the energy of (noninteracting) two light pions $\left(m_{\pi} \simeq 0.15\right.$ and $\left.0.14 \mathrm{GeV}\right)$ in our both gauge ensembles with large volumes $(L=8.1$ and $5.4 \mathrm{fm})$ is smaller than the rho meson threshold: $m_{\rho}>E_{\pi \pi}>$ $2 \sqrt{m_{\pi}^{2}+(2 \pi / L)^{2}} \approx 0.42$ and $0.54 \mathrm{GeV}$. In fact, the effective energy of $C(t)$ in Fig. 3 is clearly smaller than the rho meson resonance energy, $\simeq 770 \mathrm{MeV}$, at $t>1.2 \mathrm{fm}$.

The right panel of Fig. 1 is a relative statistical error of correlation functions for each volume, which shows that the $L / a=96$ errors are comparable with $L / a=64$, even though the number of measurements in $L / a=96$ is about 

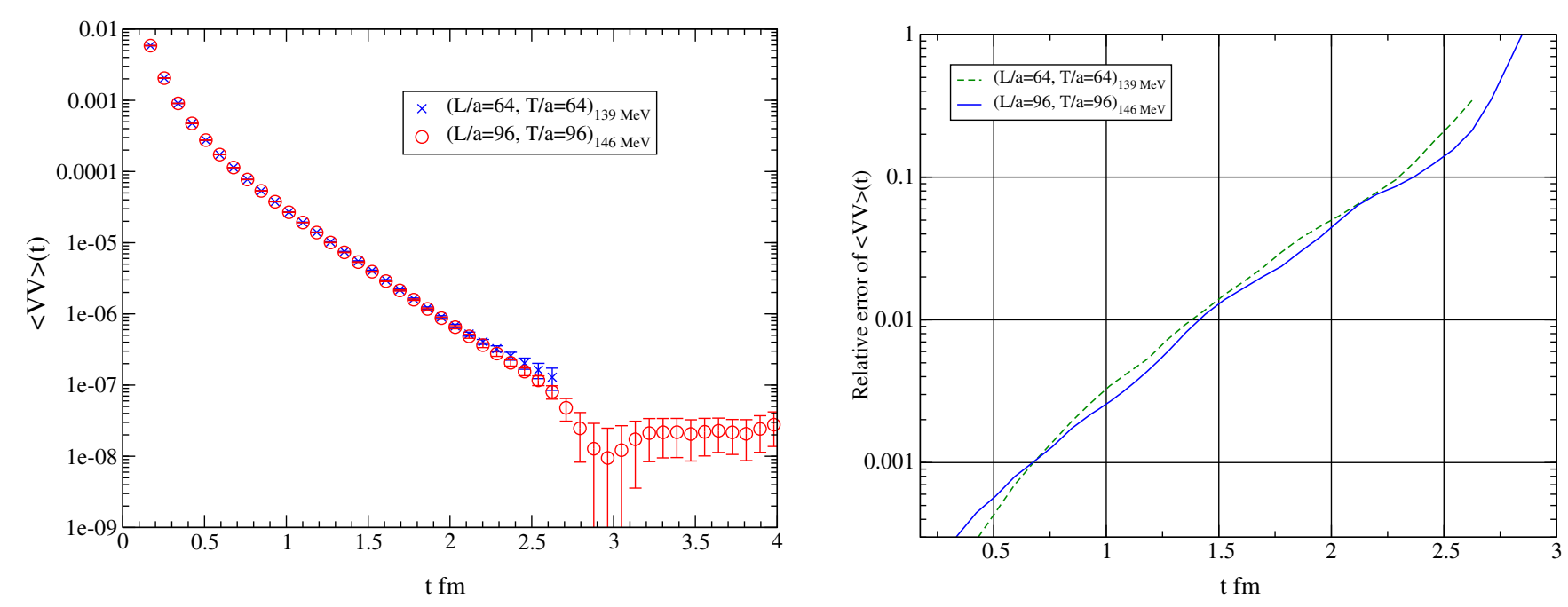

FIG. 1. (Left) The vector current two-point correlation function as a function of the time separation in fm unit. (Right) Relative error of the vector-vector correlator as a function of the time slice in fm unit. Different lines denote results of the different gauge ensembles.

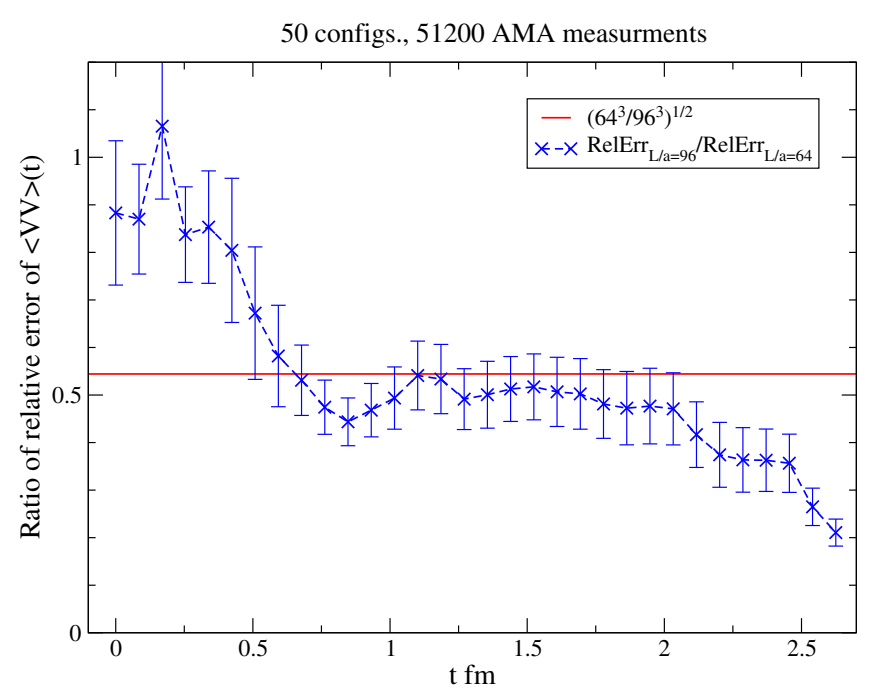

FIG. 2. The ratio of relative error of the vector-vector current correlator in $L / a=96$ and $L / a=64$ on the same number of measurements.

4 times smaller than $L / a=64$. To see more details of such an error reduction for the larger volume, we plot the ratio of the two relative errors from $L / a=96$ and $L / a=64$ for the same number of measurements, 51200 on 50 gauge configurations in Fig. 2. In this plot, the error bar is obtained from an error-of-error analysis in which we compute the standard error of error from a 10 sampling of standard deviations within 50 ensembles, i.e., splitting 10 of the ensemble errors obtained with 5 ensembles from 50 gauge ensembles. One can see that the ratio at $t$ between 0.5 and $2 \mathrm{fm}$ is close to the square root of the spatial volume ratio, $\sqrt{64^{3} / 96^{3}} \simeq 0.544$. Beyond $2 \mathrm{fm}$, the relative error further decreases due to the large statistical error of the smaller lattice $\left(64^{4}\right)$ in which the BSP becomes significant

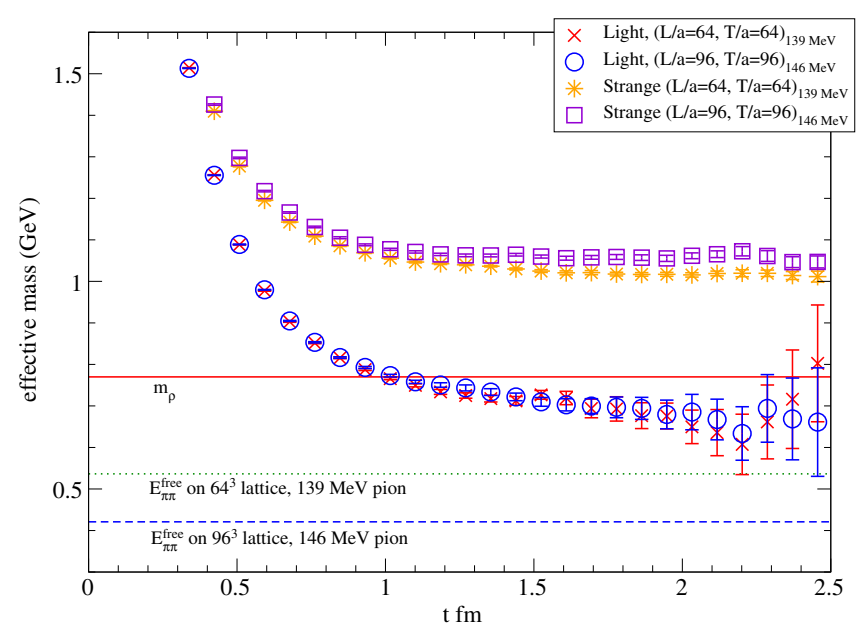

FIG. 3. The effective mass plot of the vector-vector current correlator at light and strange quark flavor. Different symbols denote the results in different flavors and lattice sizes. The straight solid line shows the experimental value of the rho meson mass, and the dashed line and dotted line show the 2 free pion energy in $m_{\pi}=0.146 \mathrm{GeV}$ on the $96^{4}$ lattice and $m_{\pi}=$ $0.139 \mathrm{GeV}$ on the $64^{4}$ lattice, respectively.

as we will discuss in the next subsection. This statistical advantage on the large lattice volume is an encouraging observation for the HVP calculation. ${ }^{2}$

\footnotetext{
${ }^{2}$ While we do not have a definite theoretical explanation as to this error reduction in large volume, the lighter pion mass for the smaller box $\left(64^{4}\right)$ would naturally cause this reduction. However, this may be the unlikely explanation of the constant behavior seen at $t \in[1,2 \mathrm{fm}]$ in Fig. 2. As studied in [50], the stability of the spectral gap in the Wilson-type fermion in large volume may be the other possibility. Further details of such an error reduction using more larger lattice sizes at exactly the same pion mass is very interesting.
} 


\section{B. Computation of $a_{\mu}^{\text {hvp }}$}

In this section we present the volume dependence for the integrand of $\left[a_{\mu}^{\mathrm{hvp}}\right]_{\text {lat }}$ [see Eq. (9)] and its time-slice summation. The integrand at time-slice $t$ and time-slice summation up to $t_{\text {cut }}$ for the light-quark contribution on each lattice volume is compared in Fig. 4. The light quark contribution $\left[a_{\mu}^{\text {hvp }}\right]_{\text {lat }}^{l}$ is dominated in $\left[a_{\mu}^{\text {hvp }}\right]_{\text {lat }}$ (the strange quark contribution to $\left[a_{\mu}^{\text {hvp }}\right]_{\text {lat }}$ is a few percent magnitude due to its larger mass and $1 / 5=\left(e_{s} / e_{l}\right)^{2}$ factor of electric charge ratio; see Sec. IV C). Compared between the $L / a=$ 96 and $L / a=64$ lattice, the shape of its integrand is similar to each other until $t=1 \mathrm{fm}$, and it then appears that $64^{4}$ lattice data are slightly larger than $96^{4}$ lattice data at $t \simeq 1.3 \mathrm{fm}$. In the right panel of Fig. 4, however, we observe that its time-slice summation is not significantly different even at $t_{\text {cut }}=2.5 \mathrm{fm}$. It means there is not so large a FV effect on $L / a=64$, which is a similar order of magnitude to its statistical fluctuation. To robustly estimate the magnitude of the FV effect between $L / a=$ 64 and $L / a=96$, we take into account the correction of its mass difference, $146 \mathrm{MeV}(L / a=96)$ and $139 \mathrm{MeV}$ pion $(L / a=64)$ as shown in Sec. V.

In order to observe the appearance of BSP into the integrand and its time-slice summation, we compare two sizes of the temporal extension on the $L / a=64$ lattice; one is the original size as $T / a=64$ and another is the extended one as $T / a=128$, in Fig. 5. From $t \approx 2.4 \mathrm{fm}$, the BSP contribution to the integrand significantly appears, and the time-slice summation is then maximally affected by BSP

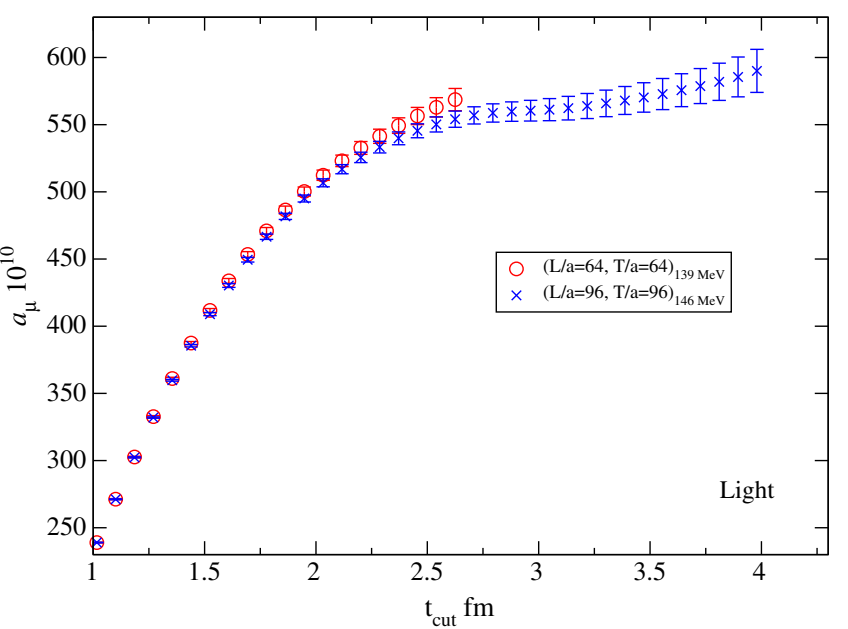

FIG. 4. (Left) Integrand of $\left[a_{\mu}^{\text {hvp }}\right]_{\text {lat }}$ in Eq. (9) divided by the lattice spacing as a function of the time slice in the physical unit. Different symbols denote the results in each gauge ensemble at light flavor. (Right) Time-slice summation for $\left[a_{\mu}^{\text {hvp }}\right]_{\text {lat }}$ up to $t_{\text {cut }}$ at the light-quark flavor. Different symbols denote the data of each gauge ensemble.
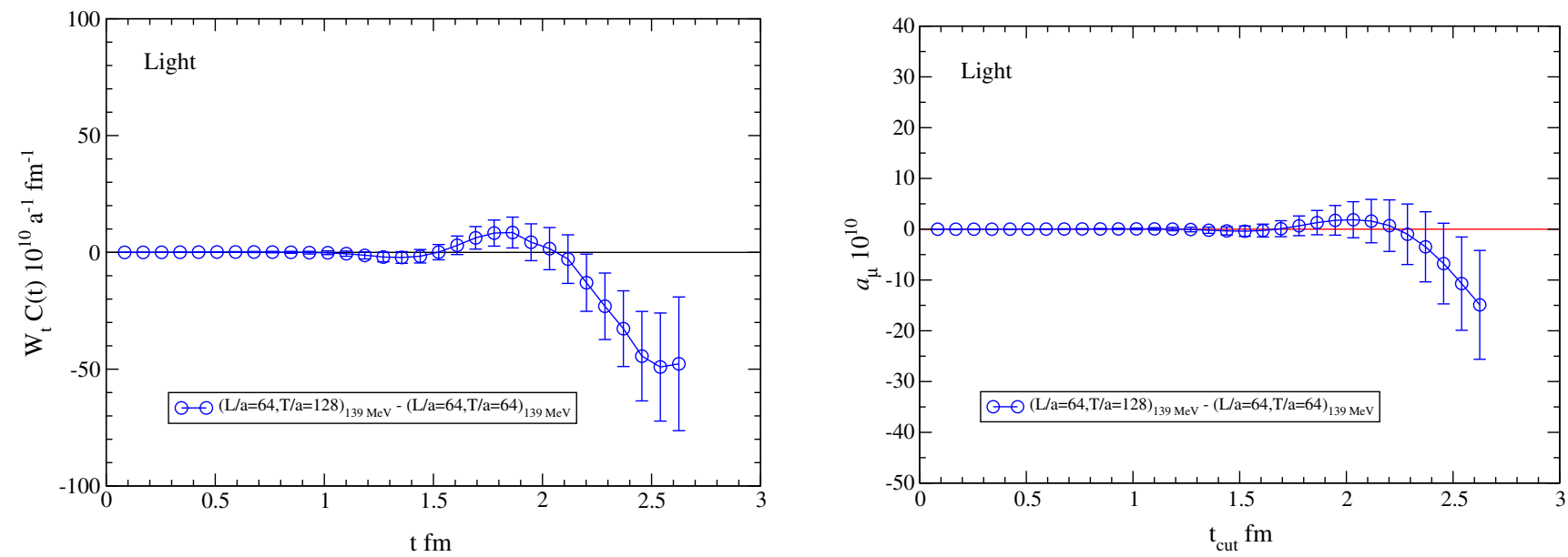

FIG. 5. Difference of the integrand divided by the lattice spacing (left) and time-slice summation up to $t_{\text {cut }}$ (right) from the $L / a=64$, $T / a=128$ lattice to the $L / a=64, T / a=64$ lattice at the light-quark flavor. 

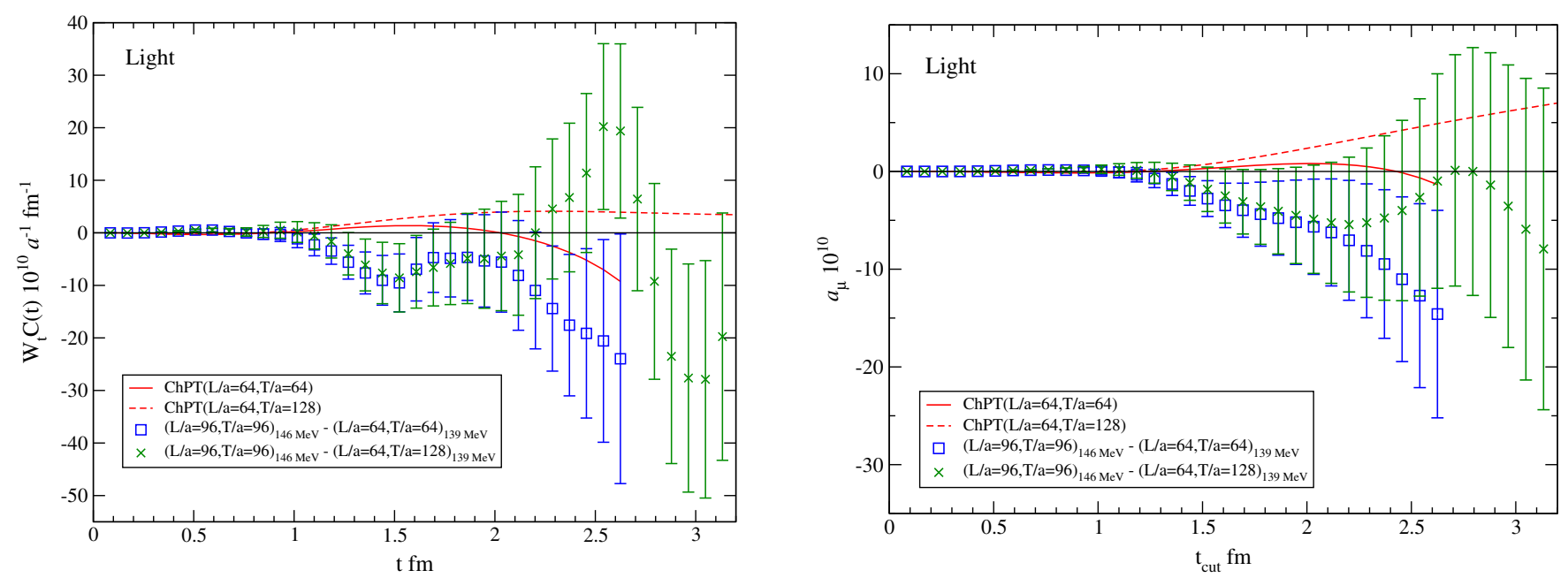

FIG. 6. (Left) Plot of the difference from the integrand on the $96^{4}$ lattice with $146 \mathrm{MeV}$ pion to the integrand on the $64^{4}$ lattice (squared symbol), and the $64^{3} \times 128$ lattice (cross symbol), with $139 \mathrm{MeV}$ pion at the light-quark flavor. (Right) The difference of $\left[a_{\mu}^{\text {hvp }}\right]$ lat up to $t_{\text {cut }}$ on different lattice sizes using the same symbol with the left panel at the light-quark flavor. Note that there has not been a mass correction between the $96^{4}$ and $64^{4}$ lattices yet. See in Sec. V. Solid (dashed) lines in both figures denote the leading order of ChPT on the $64^{4}\left(64^{3} \times 128\right)$ lattice.

at about a $4 \%$ contribution at $t_{\text {cut }}=2.6 \mathrm{fm}$. To avoid the unwanted contribution to BSP on the $T / a=64$ lattice, $t_{\text {cut }}=2 \mathrm{fm}$ is safe.

As in Fig. 6, showing the difference between $L / a=96$ and $L / a=64$ lattice at the light-quark flavor, the integrand is excellently consistent until $t=1 \mathrm{fm}$. From $t=1$ to $2 \mathrm{fm}$, a slightly negative discrepancy appears, while it is less than $10 \times 10^{-10}$ for $\left[a_{\mu}^{\text {hvp }}\right]_{\text {lat }}^{l}$ at $t_{\text {cut }} \approx 2 \mathrm{fm}$. At $t>2 \mathrm{fm}$ on $T / a=64$, since there is a significant appearance of BSP as a positive effect, which has been observed in Fig. 5, the discrepancy between the data of $T / a=64$ and $T / a=128$ also appear. Note that, as mentioned before, the discrepancy between the data of $96^{4}$ and $64^{4}$ lattice ensembles may be due, not to a FV effect but rather, a mass correction to a slight pion mass difference $\sim 7 \mathrm{MeV}$ between two ensembles. Compared to the leading order of chiral perturbation theory (ChPT)[34,44], which indicates that the integrand increases from heavy to light mass $\left(m_{\pi}=146 \mathrm{MeV} \rightarrow 139 \mathrm{MeV}\right)$, while it decreases from large to small volume $(L / a=96 \rightarrow 64)$, such a discrepancy becomes small by the cancellation of both effects. In Fig. 6 and after, we present the comparison with ChPT on the corresponding box sizes. One can see that the discrepancy of $\left[a_{\mu}^{\text {hvp }}\right]$ lat between $96^{4}$ and $64^{4}$ lattice ensembles slightly differs from the ChPT lines including those signs. Besides, it is mostly overlapping with a $1-\sigma$ statistical error bar. Later a further comparison will discuss after the analysis of the mass difference on the same volume and volume difference on the same mass with the extrapolation using mass-reweighted lattice data. Note that the ChPT line at $t>2 \mathrm{fm}$ with $T / a=64$ has negative curvature due to the BSP effect, which is consistent behavior with the lattice data.

\section{Upper and lower bound}

Here we estimate that the bound of $\left[a_{\mu}^{\mathrm{hvp}}\right]_{\text {lat }}^{l}$ should be satisfied at $t_{\text {cut }}$ as argued in $[30,31,33,51]$. The upper bound under the assumption of the free two-pion state dominance is given as

$$
\begin{aligned}
{\left[a_{\mu}^{\mathrm{hvp}}\right]_{\mathrm{upper}}=} & {\left[a_{\mu}^{\mathrm{hvp}}\right]_{\mathrm{lat}}\left(t_{\mathrm{cut}}\right) } \\
& +\sum_{t / a=t_{\mathrm{cut}} / a}^{\infty} C\left(t_{\mathrm{cut}}\right) \frac{e^{-E_{\pi \pi} t}}{e^{-E_{\pi \pi} t_{\mathrm{cut}}}+e^{-E_{\pi \pi}\left(T-t_{\mathrm{cut}}\right)}} W_{t}(t),
\end{aligned}
$$

where $E_{\pi \pi}$ represents the energy of the free two-pion state, $E_{\pi \pi}=2 \sqrt{m_{\pi}^{2}+(2 \pi / L)^{2}}$. In fact, one can see from Fig. 3 that the effective mass of the vector-vector current correlator is still above $E_{\pi \pi}$ even at $t>1.5 \mathrm{fm}$, and so that using the integrand switched to the single exponential function with $E_{\pi \pi}$ after $t=t_{\text {cut }}$, Eq. (14) is a restricted upper bound for $a_{\mu}^{\text {hvp }}$ in the time-slice summation. On the other hand, the lower bound we take is two forms,

$$
\begin{aligned}
& {\left[a_{\mu}^{\mathrm{hvp}}\right]_{\mathrm{lower}(0)}=\left[a_{\mu}^{\mathrm{hvp}}\right]_{\text {lat }}\left(t_{\mathrm{cut}}\right), } \\
{\left[a_{\mu}^{\mathrm{hvp}}\right]_{\mathrm{lower}\left(\mathrm{m}_{\rho}\right)}=} & {\left[a_{\mu}^{\mathrm{hvp}}\right]_{\mathrm{lat}}\left(t_{\mathrm{cut}}\right) } \\
& +\sum_{t / a=t_{\mathrm{cut}} / a}^{\infty} C\left(t_{\mathrm{cut}}\right) \frac{e^{-m_{\rho} t}}{e^{-m_{\rho} t_{\mathrm{cut}}}+e^{-m_{\rho}\left(T-t_{\mathrm{cut}}\right)}} W_{t}(t) .
\end{aligned}
$$

The first lower bound in Eq. (15) is consistent with $\left[a_{\mu}^{\text {hvp }}\right]_{\text {lat }}$ since we know the remnant integral from $t_{\text {cut }}$ to infinity is a positive contribution. Otherwise, the second lower bound in 

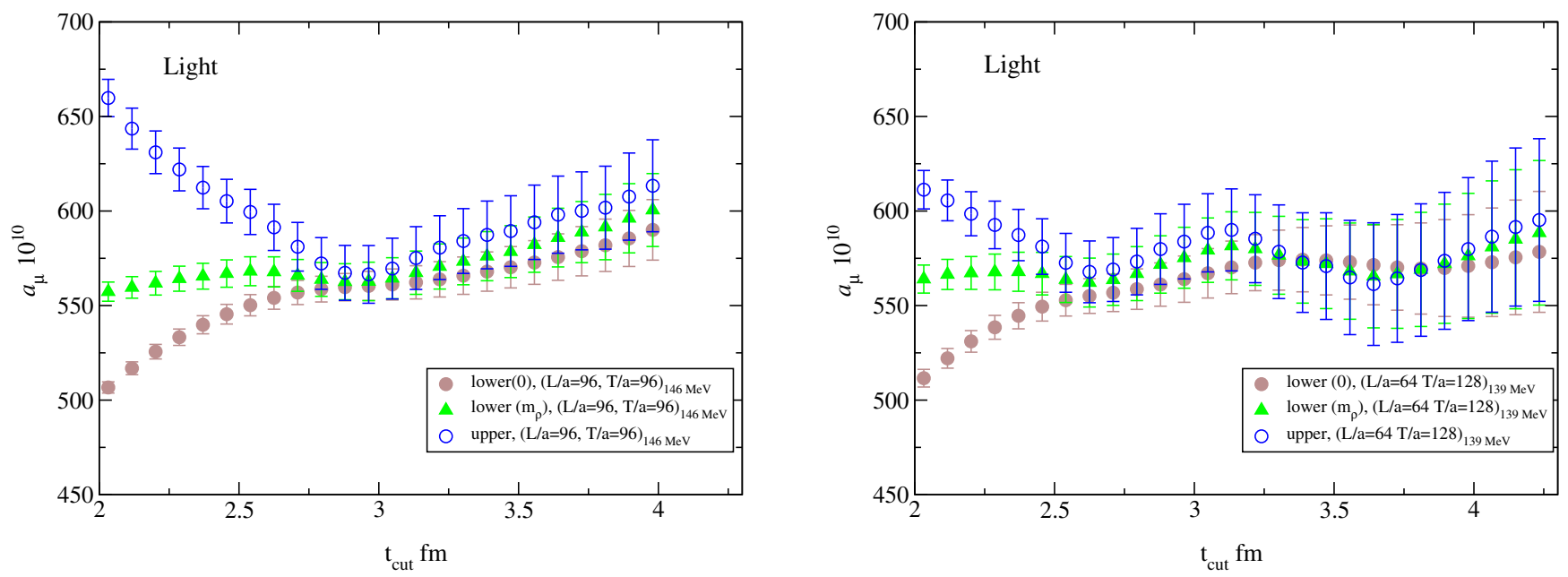

FIG. 7. This plot shows the upper and lower bounds of $\left[a_{\mu}^{\text {hvp }}\right]_{\text {lat }}^{l}$ at each $t_{\text {cut }}$ in the light-quark flavor on the $96^{4}$ lattice (left) and the $64^{3} \times 128$ lattice (right). The filled circle and triangle symbols denote the lower-bound defined in Eqs. (15) and (16), respectively. The open cross symbol denotes the upper bound in Eq. (14).

Eq. (16) consists of the exponential function with the rho mass $(0.775 \mathrm{GeV})$ from $t>t_{\text {cut }}$, which is the same form as the upper bound of Eq. (14) instead of $E_{\pi \pi}$. The second one is a more restricted bound, since an additional contribution of the rho state is taken into account. Figure 3 which presents the lower exponent of the vector-vector current correlator than the rho mass at $t>1 \mathrm{fm}$ actually shows that such a restricted lower bound is reasonable for our data.

Figure 7 shows such a lower and upper bound on two lattice volumes, $L / a=96$ and $L / a=64$ lattices. At $t_{\text {cut }} \approx 3 \mathrm{fm}$, two bounds become consistent within $1 \sigma$ statistical error. Since the statistical error of upper-bound is larger than two lower-bounds due to rather large fluctuation of $C\left(t_{\text {cut }}\right)$, the upper-bound at $t_{\text {cut }} \approx 3.0 \mathrm{fm}$ is regarded as a possible range of $\left[a_{\mu}^{\text {hvp }}\right]^{\text {light }}$. In our analysis, we have

$$
\begin{aligned}
554 & <\left[a_{\mu}^{\text {hvp }}\right]_{\text {lat }}^{l} \times 10^{10}<586, \\
{[L / a} & =96 \text { lattice with } 146 \mathrm{MeV} \text { pion }], \\
562 & <\left[a_{\mu}^{\text {hvp }}\right]_{\text {lat }}^{l} \times 10^{10}<609, \\
{[L / a} & =64 \text { lattice with } 139 \mathrm{MeV} \text { pion }],
\end{aligned}
$$

in which the upper value of $\left[a_{\mu}^{\text {hvp }}\right]_{\text {upper }}^{l}$ and the lower value of $\left[a_{\mu}^{\mathrm{hvp}}\right]_{\text {lower }\left(\mathrm{m}_{\rho}\right)}^{l}$ are consistent within 1- $\sigma$ statistical error. One can see two regions are mostly overlapping.

\section{Strange quark contribution}

Since our gauge ensembles have the different sea strange mass on $96^{4}$ and $64^{4}$ (see in Table I), the strange quark contribution to muon $g-2$ should be more significant due to its mass correction than the FV effect. In Fig. 8, we plot both data of $\left[a_{\mu}^{\mathrm{hvp}}\right]_{\text {lat }}^{s}$ and the integrand of the $Q^{2}$ integral for

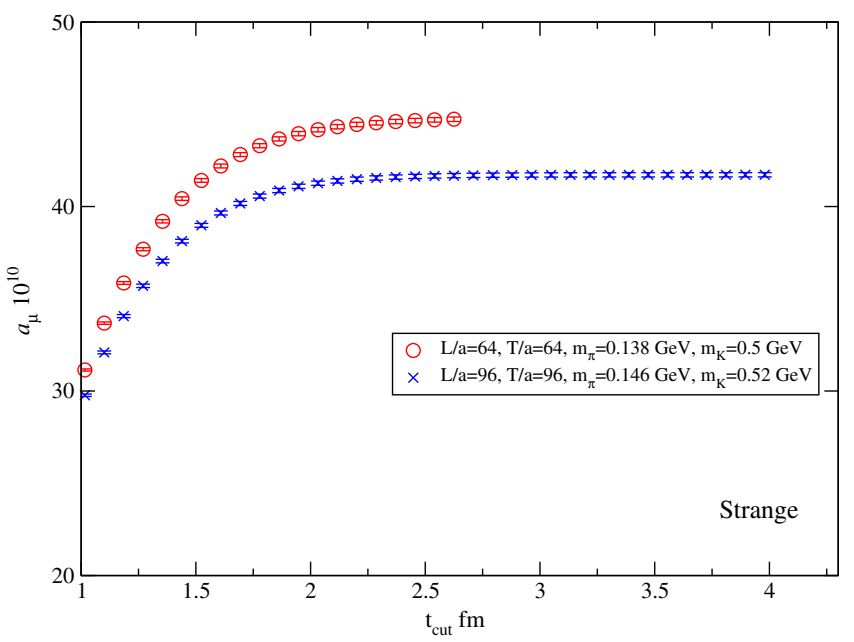

FIG. 8. The time-slice summation of $\left[a_{\mu}^{\mathrm{hvp}}\right]_{\text {lat }}^{s}$ up to $t_{\text {cut }}$ at the strange quark flavor.

a comparison in the strange sector. One can see that $\left[a_{\mu}^{\text {hvp }}\right]_{\text {lat }}^{s}$ on $96^{4}$ is $6-7 \%$ smaller than that on $64^{4}$ lattice, besides, a contribution of such a discrepancy to the total muon $g-2$ is minor. Actually its magnitude is less than $0.5 \%$.

\section{MASS CORRECTION AND FINITE VOLUME EFFECT}

As mentioned before, on the $96^{4}$ lattice ensemble there is $\approx 7 \mathrm{MeV}$ pion mass difference from the $64^{4}$ lattice which will be affected in the FV effect of $\left[a_{\mu}^{\text {hvp }}\right]_{\text {lat }}^{l}$, and so that to estimate such an effect we compare $\left[a_{\mu}^{\text {hvp }}\right]_{\text {lat }}^{l}$ and its integrand with the shifted sea quark mass as well as the valence quark by reweighting onto $135 \mathrm{MeV}$ pion on the $64^{4}$ lattice (see those lattice parameters in Table I). 

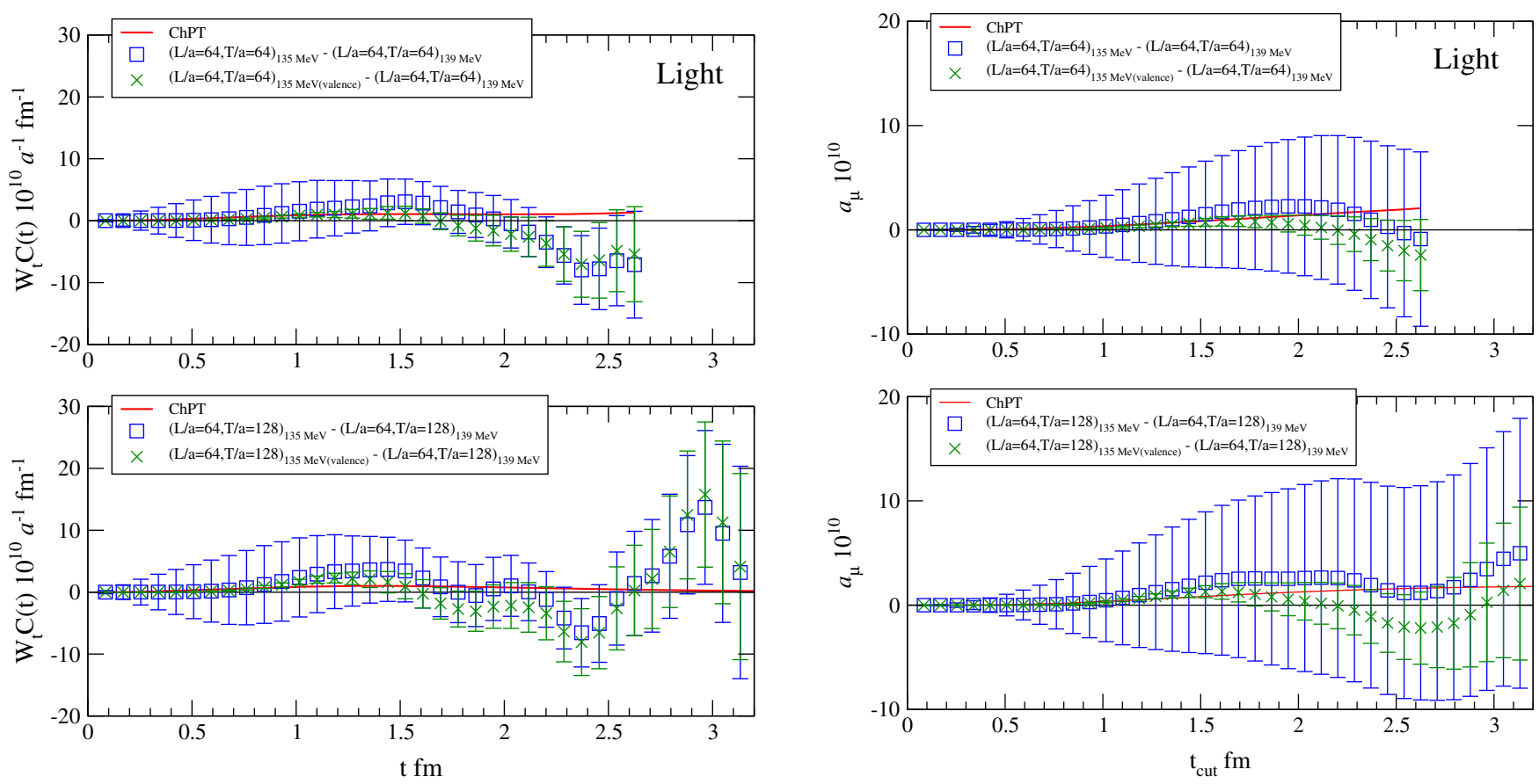

FIG. 9. (Left) The difference of the integrand between $135 \mathrm{MeV}$ pion data using the reweighting method and $139 \mathrm{MeV}$ pion data on $L / a=64$. The top and bottom panels present the comparison with the partially quenched case and extended temporal extension, respectively. (Right) The symbols are same as the left panel for $\left[a_{\mu}^{\text {hvp }}\right]_{\text {lat }}^{l}$ up to $t_{\text {cut }}$. Solid (dashed) lines denote the leading order of ChPT with $T / a=64(T / a=128)$.

As shown in Fig. 9, the correction of different pion masses is as a slightly positive shift of $\left[a_{\mu}^{\text {hvp }}\right]_{\text {lat }}^{l}$ and its integrand also increases when the pion mass decreases. Compared to the partially quenched case, the error of the reweighting factor becomes the dominant contribution, especially for a short time slice, while in the long time slice its error is comparable with each other since statistical fluctuation is large in both cases. At large time slices over $t=2 \mathrm{fm}$ on $T / a=64$, one can see that the BSP effect significantly appears in the comparison with the extended temporal length $T / a=128$. The mass correction to $\left[a_{\mu}^{\text {hvp }}\right]_{\text {lat }}^{l}$ is evaluated as $(4 \pm 12) \times$ $10^{-10}$ referred to Fig. 9 at $t_{\text {cut }}=3 \mathrm{fm}$ in the $64^{3} \times 128$ lattice. Compared to the leading order of ChPT, such a mass correction to $\left[a_{\mu}^{\text {hvp }}\right]_{\text {lat }}^{l}$ is consistent with lattice data for both the integrand and its time-slice summation within 1- $\sigma$ statistical error even in the short time slice.

As presented in [32], they showed the strong growth of $a_{\mu}^{\text {hvp }}$ when $m_{\pi}^{2}$ decreases. When naively applying the linear $m_{\pi}^{2}$ behavior for their $a_{\mu}^{\text {hvp }}$ values $^{3}$ between $m_{\pi}=0.19 \mathrm{GeV}$ and $m_{\pi}=0.135 \mathrm{GeV}$, a decrease of $6 \%$ pion mass affects roughly a $7 \times 10^{10}$ positive contribution to $\left[a_{\mu}^{\text {hvp }}\right]_{\text {lat }}$ at the

\footnotetext{
${ }^{3}$ In Ref. [32], they have showed only combined results with several ansatz to perform the integral from $t_{\text {cut }}$ to infinity. Since their $t_{\text {cut }}$ is much smaller than ours, this estimate of mass dependence for $a_{\mu}^{\text {hvp }}$ is naive.
}

light-quark flavor. This value is roughly the same magnitude as our estimate.

To estimate the FV correction between $96^{4}$ and $64^{4}$ at the same pion mass, we first evaluate linearly extrapolated data into $146 \mathrm{MeV}$ pion on the $L / a=64$ lattice ensemble using two data of $139 \mathrm{MeV}$ pion and $135 \mathrm{MeV}$ pion, and we then take the difference between $L / a=96$ and $L / a=64$ in $146 \mathrm{MeV}$ pion. In Fig. 10, we show such a comparison with a different volume. One can see that the difference between data on the $64^{4}$ lattice in $139 \mathrm{MeV}$ pion and the $96^{4}$ lattice in $146 \mathrm{MeV}$ pion is canceled by the contribution of a mass correction in Fig. 9, and as this result, the FV effect is consistently zero within the statistical error. Conservatively, we regard the FV correction as the volume difference at $t_{\text {cut }}=3 \mathrm{fm}$ of the cross-symbol in Fig. 10 where the BSP effect is negligible and $t_{\text {cut }}$ dependence is minor. The magnitude of FV correction to $\left[a_{\mu}^{\text {hvp }}\right]_{\text {lat }}^{l}$ on $L=5.4 \mathrm{fm}$ is then $(10 \pm 26) \times 10^{-10}$, which corresponds to $1 \pm 4 \%$ for the dispersive estimate $a_{\mu}^{\text {hvp }} \approx 700 \times 10^{-10}$. We also plot the ChPT line in Fig. 6. Although the lattice estimate is still consistent with ChPT within 1- $\sigma$ statistical error, the central value on both lattice sizes is slightly over the ChPT line. To do a more clear comparison, high statistics data on larger lattice size than $L=8 \mathrm{fm}$ at exactly same quark mass as $64^{4}$ lattice ensemble are needed, in particular for the infrared region. 

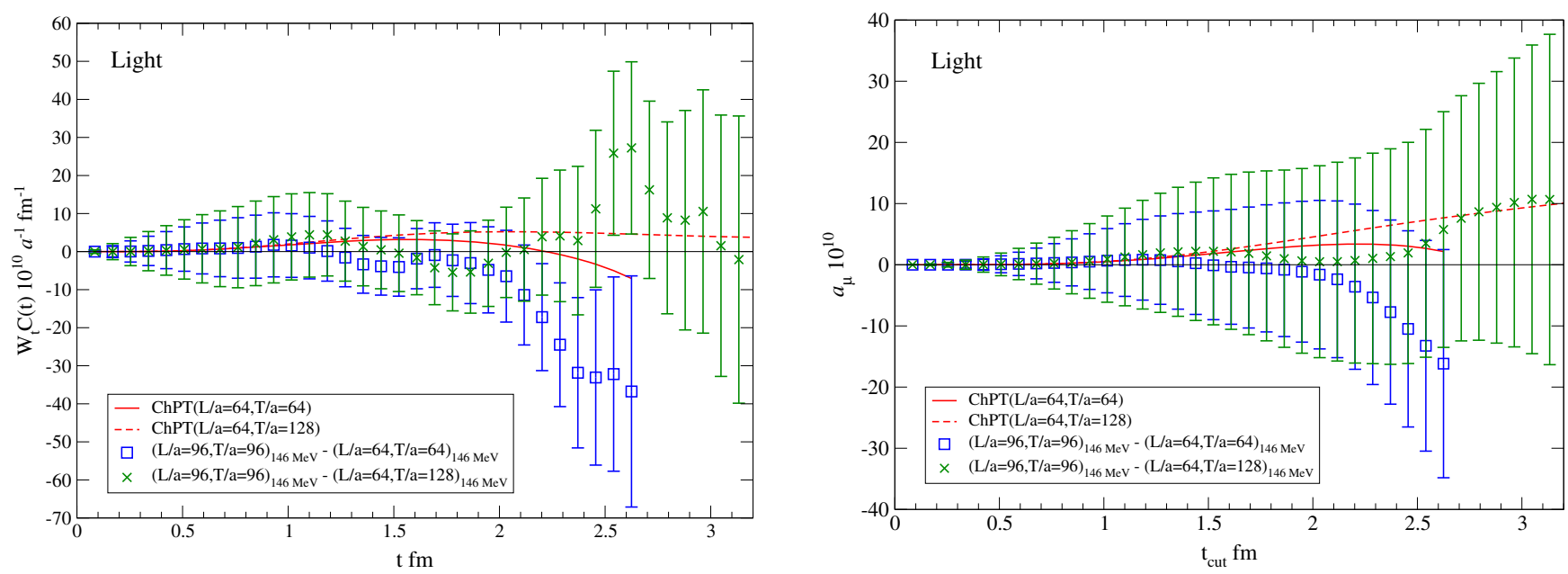

FIG. 10. The finite-volume effect of the integrand (left) and $\left[a_{\mu}^{\text {hvp }}\right]_{\text {lat }}^{l}$ up to $t_{\text {cut }}$ (right) with a mass correction to Fig. 6 estimated from Fig. 9. Solid (dashed) lines denote the leading order of ChPT with $T / a=64^{4}(T / a=128)$.

\section{SUMMARY}

This paper presents the study of the FV correction for the connected diagram of the hadronic vacuum polarization contribution to muon $g-2$ from the direct comparison with two volumes, $L=5.4$ and $8.1 \mathrm{fm}$, in purely lattice QCD, which is an independent way from the other lattice studies [29,31,33]. At the physical pion, we estimate the FV correction in the TMR method using the time-slice summation of the vector-vector current correlator. Using the high-statistics lattice data boosted by the AMA method, we obtain that the light-quark contribution to $\left[a_{\mu}^{\text {hvp }}\right]_{\text {lat }}$ estimated in the time-slice summation on $L=5.4 \mathrm{fm}$ is the $(10 \pm$ 26) $\times 10^{-10}$ shift from $L=8.1 \mathrm{fm}$ as the FV correction at $146 \mathrm{MeV}$ pion, correspondingly, the $1 \% \pm 4 \%$ effect to the dispersive estimate of $a_{\mu}^{\text {hvp }} \approx 700 \times 10^{-10}$ and $\left[a_{\mu}^{\text {hvp }}\right]_{\text {lat }}$ taken as the upper and lower bound in Eq. (17) is obtained in Eqs. (14) and (16). In our study, compared to the estimate of the leading order of ChPT, there is no observation of a significant discrepancy and it is then consistent within 1- $\sigma$ statistical error, although the statistical fluctuation is still large. Here we also have a concern of the uncertainty due to the truncation of the integral in TMR using finite $t_{\text {cut }}$, in which we expect that the FV correction becomes significantly large after $t_{\text {cut }}$. In order to completely remove such an uncertainty, the infinite volume limit at the physcal pion is necessary to realize $\left[a_{\mu}^{\text {hvp }}\right]_{\text {lat }}$ at $t_{\text {cut }} \rightarrow \infty$ in TMR. This will be done by using one more large lattice ensemble generated by the PACS Collaboration in the future. Our approach is also useful to check the estimate of the FV correction that relied on the extrapolation into the physical pion and infinite volume limit simultaneously using lattice data with various pion masses and volumes [29,31,32,52].

Furthermore there are several systematics which have not been taken into account. First, since there is only one lattice cutoff scale on this ensemble, the lattice artifact effect involved in $a_{\mu}^{\text {hvp }}$ cannot be measured directly. We try to partially estimate it by comparison with the representation of $\left[\tilde{a}_{\mu}^{\text {hvp }}\right]_{\text {lat }}$ in Eq. (11). On such a way, the lattice artifact correction appears in the short time slice, especially at $t / a=1$, and for the integral it thus affects the constant shift. The magnitude of the shift for $\left[a_{\mu}^{\text {hvp }}\right]_{\text {lat }}^{l}$ at $t_{\text {cut }}=$ $3.02 \mathrm{fm}$ is $\left(\left[\tilde{a}_{\mu}^{\text {hvp }}\right]_{\text {lat }}^{l}-\left[a_{\mu}^{\text {hvp }}\right]_{\text {lat }}^{l}\right) \times 10^{10}=9.11(1)$ which is a roughly $2 \%$ effect for the total light flavor contribution. Note that this difference is only a consequence of discretized space-time on finite lattice spacing. The other lattice artifact caused by chiral symmetry breaking in the Wilson-clover fermion should be estimated in the future using a larger cutoff scale. Second, as mentioned before, this is a calculation of the only connected diagram, and the disconnected piece as the SU(3) flavor symmetry breaking in the electromagnetic current is the other missing factor in our analysis. Although several papers $[30,32,53]$ for the computation of the disconnected piece in the lattice QCD have reported a negative contribution to $a_{\mu}^{\text {hvp }}$ as $1.5 \%$, it will be tested on this ensemble in the next work.

The future generation of several gauge ensembles with one more large volume and fine lattice spacing in the PACS Collaboration enables us to provide the final result in lattice QCD by simultaneously taking the infinite volume and continuum limit.

\section{ACKNOWLEDGMENTS}

We would like to thank the PACS, RBC-UKQCD Collaboration for helpful discussions and support. T. I. and C.L. are supported in part by U.S. DOE Contract No. DESC0012704 (BNL). T. I. is also supported by JSPS KAKENHI Grants No. JP26400261 and No. JP17H02906. C. L. is also supported by a DOE Office of Science Early Career Award. We originally developed the computation code based on the Columbia Physics System (CPS) in 
which the ztuned OpenQCD system (http://luscher.web .cern.ch/luscher/openQCD/) is embedded. This work is supported in part by MEXT as "Priority Issue on Post-K computer" (Education of the Fundamental Laws and Evolution of the Universe) and Joint Institute for Computational Fundamental Science (JICFuS), and the U.S.-Japan Science and Technology Cooperation Program in High Energy Physics for FY2018. Numerical calculations were performed on the $\mathrm{K}$ computer in the RIKEN Center for Computational Science (CCS), Hokusai at the Advanced Center for Computing and Communication (ACCC) in RIKEN, XC40 at YITP at the Kyoto University, BlueGene/Q in the High Energy Accelerator Research Organization (KEK). The computation was also carried out using the computer facilities at the Research Institute for Information Technology, Kyushu University, and conducted using the Fujitsu PRIMERGY CX600M1/ CX1640M1 (Oakforest-PACS) in the Information Technology Center, The University of Tokyo. This computation is also supported by the Interdisciplinary Computational Science Program No. xg17i019, No. xg18i015 in Tsukuba CCS, Large Scale Simulation Program No. 16/17-26 in KEK, General use No. G17029, No. G18001 at ACCC, and resources of the K computer provided by the RIKEN-CCS through the High Performance Computing Infrastructure (HPCI) System Research project (Project ID:hp180126).

\section{APPENDIX: PERFORMANCE TEST OF AMA WITH SAP DEFLATION}

In this Appendix, we present the numerical test of AMA performance with two deflation methods, low-mode deflation and SAP deflation. For low-mode deflation, we compute the single-precision low mode of the Wilsonclover kernel by the Lanczos procedure with Chebychev acceleration (see in [39]). On $96^{4}$ PACS configurations, we have 750 low modes as $10^{-8}$ accuracy, and the conjugate gradient (CG) method is used to obtain $\mathcal{O}^{\text {(org) }}$ and $\mathcal{O}^{\text {(appx) }}$. Its approximation, having the similar correlation, to be $\Delta_{r} \simeq O\left(10^{-4}\right)$, is generated by $600 \mathrm{CG}$ iteration with lowmode projection. Using SAP deflation, the approximation is obtained with the same parameters as in Table II.

From Fig. 11, one can clearly see the computational cost for the generation of a deflation field is much reduced by a factor 70, and also the cost of the quark propagator (12 times iterative solver is performed) using GCR with SAP deflation is 9 times for exact and 3 times for an approximation smaller than the case using $600 \mathrm{CG}$ iteration with a low-mode deflation. In total, the computational time of AMA with SAP deflation is reduced by a factor 3 and more. We note that, for low-mode deflation, once we obtain the low-mode vector, it enables us to recycle this data by loading it from disk storage to construct the low-mode projection matrix without the additional cost of the little Dirac solver during the iterative process as in the SAP

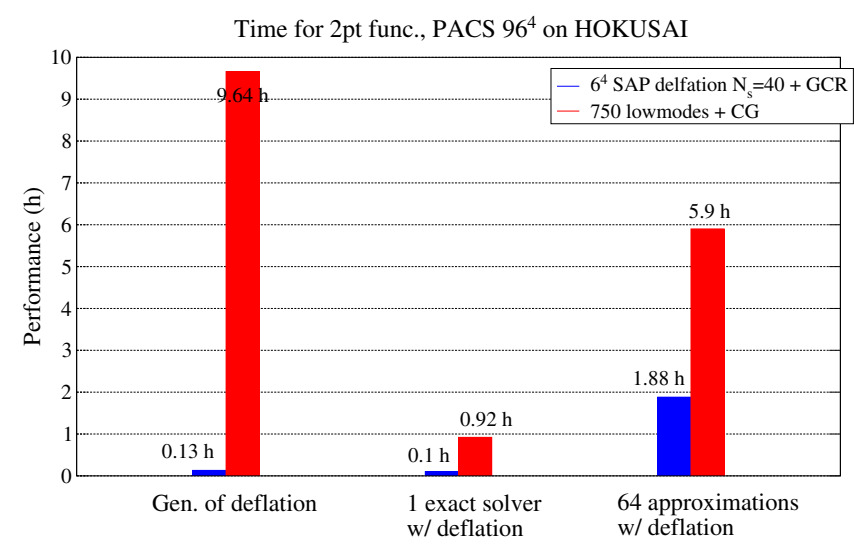

FIG. 11. The comparison of elapsed time in three cases for the AMA process; generation of the deflation field, computation of a quark propagator in exact precision $10^{-10}$ of residual norm, and in the approximation. In the approximation, we compare the computational time for $N_{G}=64$, which is the number of different source locations. We perform this test on HOKUSAI, in which the Fujitsu FX100 CPU chips having 32 cores per node are equipped. The SAP deflation result is on 128 nodes, while the low-mode deflation result is used as the scaled one from 256 nodes to 128 nodes assuming the strong scaling.

deflation [49]; however, for the large size of the lattice, as we demonstrated in $96^{4}$, storing 750 eigenmodes, 6 TB disk space is needed per configuration. It turns out to be a diskconsumed scheme. Furthermore, increasing the lattice size, since the number of the low-lying eigenmodes densely increase near zero, the low-mode deflation computed by the Lanczos algorithm will require a huge computation

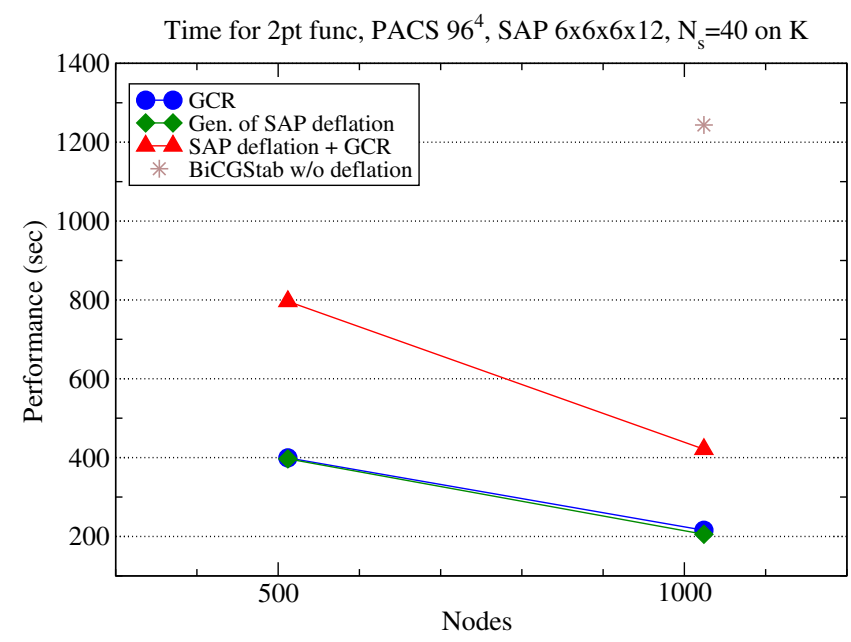

FIG. 12. This plot is a strong scaling test of SAP deflation + GCR on the PACS $96^{4}$ lattice. Here the SAP block size is $6^{3} \times 12$ with $N_{s}=40$ SAP deflation fields. We carry out a computation of the quark propagator on the K computer, in which the Fujitsu FX10 chip having 8 cores per node is equipped. The flat MPI run is used in this test. For comparison, we plot the computational time in the BiCGstab method without deflation as the star symbol. Note that the star symbol is scaled from 2048 nodes under the assumption of strong scaling. 
resource, for instance large memory size and disk space to store eigenmodes.

On the other hand, SAP deflation has a totally negligible cost for the generation of the deflation field, and it thus does not need to store the deflation field into disk space instead, the computation of the deflation field at each time occurs before doing the quark solver. It has an advantage to reduce the space of disk storage. In addition, as pointed out in [36], since SAP deflation can use $N_{s}$ local deflation fields by the domain decomposition of the Dirac operator onto SAP block size, the total memory size to store the deflation field is reduced by $O(10)$. This is also an advantage to reduce the requirement of the memory size.

Figure 12 shows the strong scaling of SAP deflation + GCR on the K computer accommodated in RIKEN-CCS. One can see the performance for both the generation of the SAP deflation field and GCR where the deflation projection has the strong scaling from 512 nodes to 1024 nodes. We also compare the performance of SAP deflation + GCR with the conventional method, which is the BiCGStab solver without deflation used in [47]. Even including the overhead to generate the deflation field, SAP deflation + GCR has more than 3 times better performance than the conventional method. In the measurement, $O\left(10^{3}\right)$ two-point functions per configuration are needed, so that the elapsed time of the quark solver is eventually dominated. Ignoring the time for the generation of the deflation field, the GCR with SAP deflation projection can gain 6 times speed-up. Furthermore, AMA can reduce such a solver time by a factor 5 and more, and it thus gains more than 30 times speed-up compared to the conventional one.
[1] G. W. Bennett et al. (Muon (g-2) Collaboration), Phys. Rev. Lett. 92, 161802 (2004).

[2] G. W. Bennett et al. (Muon (g-2) Collaboration), Phys. Rev. D 73, 072003 (2006).

[3] T. Aoyama, M. Hayakawa, T. Kinoshita, and M. Nio, Phys. Rev. Lett. 109, 111807 (2012).

[4] D. Babusci et al. (KLOE Collaboration), Phys. Lett. B 720, 336 (2013).

[5] D. Babusci et al. (KLOE Collaboration), Phys. Lett. B 718, 910 (2013).

[6] J. P. Lees et al. (BABAR Collaboration), Phys. Rev. D 86, 032013 (2012).

[7] M. Ablikim et al. (BESIII Collaboration), Phys. Lett. B 753, 629 (2016).

[8] K. Ackerstaff et al. (OPAL Collaboration), Eur. Phys. J. C 7, 571 (1999).

[9] S. Anderson et al. (CLEO Collaboration), Phys. Rev. D 61, 112002 (2000).

[10] S. Schael et al. (ALEPH Collaboration), Phys. Rep. 421, 191 (2005).

[11] M. Fujikawa et al. (Belle Collaboration), Phys. Rev. D 78, 072006 (2008).

[12] M. Davier, A. Hoecker, B. Malaescu, and Z. Zhang, Eur. Phys. J. C 71, 1515 (2011); 72, 1874(E) (2012).

[13] K. Hagiwara, R. Liao, A. D. Martin, D. Nomura, and T. Teubner, J. Phys. G 38, 085003 (2011).

[14] M. Davier, A. Hoecker, B. Malaescu, and Z. Zhang, Eur. Phys. J. C 77, 827 (2017).

[15] A. Keshavarzi, D. Nomura, and T. Teubner, Phys. Rev. D 97, 114025 (2018).

[16] F. Jegerlehner and A. Nyffeler, Phys. Rep. 477, 1 (2009).

[17] D. Flay (Muon (g-2) Collaboration), Proc. Sci. ICHEP2016 (2017) 1075.

[18] K. Shimomura, Proceedings, 5th International Conference on Exotic Atoms and Related Topics (EXA2014): Vienna, Austria, 2014, [Hyperfine Interact. 233, 89 (2015)].
[19] C. Patrignani et al. (Particle Data Group Collaboration), Chin. Phys. C 40, 100001 (2016).

[20] F. Jegerlehner, Proceedings, Workshop on Flavour changing and conserving processes 2015 (FCCP2015): Anacapri, Capri Island, Italy, 2015 [EPJ Web Conf. 118, 01016 (EDP Sciences, Les Ulis, 2016)].

[21] M. Gourdin and E. De Rafael, Nucl. Phys. B10, 667 (1969).

[22] T. Blum, Phys. Rev. Lett. 91, 052001 (2003).

[23] M. Gockeler, R. Horsley, W. Kurzinger, D. Pleiter, P. E. L. Rakow, and G. Schierholz (QCDSF Collaboration), Nucl. Phys. B688, 135 (2004).

[24] X. Feng, K. Jansen, M. Petschlies, and D. B. Renner, Phys. Rev. Lett. 107, 081802 (2011).

[25] P. Boyle, L. Del Debbio, E. Kerrane, and J. Zanotti, Phys. Rev. D 85, 074504 (2012).

[26] M. Della Morte, B. Jager, A. Juttner, and H. Wittig, J. High Energy Phys. 03 (2012) 055.

[27] F. Burger, X. Feng, G. Hotzel, K. Jansen, M. Petschlies, and D. B. Renner (ETM Collaboration), J. High Energy Phys. 02 (2014) 099.

[28] B. Chakraborty, C. T. H. Davies, G. C. Donald, R. J. Dowdall, J. Koponen, G. P. Lepage, and T. Teubner (HPQCD Collaboration), Phys. Rev. D 89, 114501 (2014).

[29] B. Chakraborty, C. T. H. Davies, P. G. de Oliviera, J. Koponen, and G. P. Lepage, Phys. Rev. D 96, 034516 (2017).

[30] S. Borsanyi, Z. Fodor, T. Kawanai, S. Krieg, L. Lellouch, R. Malak, K. Miura, K. K. Szabo, C. Torrero, and B. Toth, Phys. Rev. D 96, 074507 (2017).

[31] S. Borsanyi et al. (Budapest-Marseille-Wuppertal Collaboration), Phys. Rev. Lett. 121, 022002 (2018).

[32] M. Della Morte, A. Francis, V. Glpers, G. Herdoza, G. von Hippel, H. Horch, B. Jger, H. B. Meyer, A. Nyffeler, and H. Wittig, J. High Energy Phys. 10 (2017) 020.

[33] T. Blum, P. A. Boyle, V. Glpers, T. Izubuchi, L. Jin, C. Jung, A. Jttner, C. Lehner, A. Portelli, and J. T. Tsang, Phys. Rev. Lett. 121, 022003 (2018). 
[34] C. Aubin, T. Blum, P. Chau, M. Golterman, S. Peris, and C. Tu, Phys. Rev. D 93, 054508 (2016).

[35] J. Bijnens and J. Relefors, J. High Energy Phys. 12 (2017) 114.

[36] G. von Hippel, T. D. Rae, E. Shintani, and H. Wittig, Nucl. Phys. B914, 138 (2017).

[37] T. Blum, T. Izubuchi, and E. Shintani, Phys. Rev. D 88, 094503 (2013).

[38] T. Blum, T. Izubuchi, and E. Shintani, Proc. Sci. LATTICE2012 (2012) 262.

[39] E. Shintani, R. Arthur, T. Blum, T. Izubuchi, C. Jung, and C. Lehner, Phys. Rev. D 91, 114511 (2015).

[40] B. Lautrup, A. Peterman, and E. de Rafael, Phys. Rep. 3, 193 (1972).

[41] D. Bernecker and H. B. Meyer, Eur. Phys. J. A 47, 148 (2011).

[42] K. I. Ishikawa, N. Ishizuka, Y. Kuramashi, Y. Nakamura, Y. Namekawa, Y. Taniguchi, N. Ukita, T. Yamazaki, and T. Yoshi (PACS Collaboration), Proceedings, 33rd International Symposium on Lattice Field Theory (Lattice 2015): Kobe, Japan, 2015 [Proc. Sci., LATTICE2015 (Sissa Medialab srl Partita, 2016) 271]. DOI: 10.22323/1.251.0271

[43] E. Shintani, S. Aoki, H. Fukaya, S. Hashimoto, T. Kaneko, T. Onogi, and N. Yamada, Phys. Rev. D 82, 074505 (2010).

[44] M. Golterman, K. Maltman, and S. Peris, Phys. Rev. D 95, 074509 (2017).
[45] Y. Kuramashi, Hadron spectroscopy and interactions, in LATTICE2017 conference, Granada, Spain in June 2017 (unpublished).

[46] A. Hasenfratz, R. Hoffmann, and S. Schaefer, Phys. Rev. D 78, 014515 (2008).

[47] K. I. Ishikawa, N. Ishizuka, Y. Kuramashi, Y. Nakamura, Y. Namekawa, Y. Taniguchi, N. Ukita, T. Yamazaki, and T. Yoshie (PACS Collaboraiton), Proceedings, 33rd International Symposium on Lattice Field Theory (Lattice 2015): Kobe, Japan, 2015, [Proc. Sci., LATTICE2015 (Sissa Medialab srl Partita, 2016) 075]. DOI: 10.22323/ 1.251 .0075

[48] M. Luscher, Comput. Phys. Commun. 156, 209 (2004).

[49] M. Luscher, J. High Energy Phys. 07 (2007) 081.

[50] L. Del Debbio, L. Giusti, M. Luscher, R. Petronzio, and N. Tantalo, J. High Energy Phys. 02 (2006) 011.

[51] C. Lehner, The hadronic vacuum polarization contribution to the muon anomalous magnetic moment, RBRC Workshop on Lattice Gauge Theories, 2016 (unpublished).

[52] D. Giusti, V. Lubicz, G. Martinelli, F. Sanfilippo, and S. Simula, J. High Energy Phys. 10 (2017) 157.

[53] T. Blum, P. A. Boyle, T. Izubuchi, L. Jin, A. Jttner, C. Lehner, K. Maltman, M. Marinkovic, A. Portelli, and M. Spraggs, Phys. Rev. Lett. 116, 232002 (2016). 